TITLE:

\title{
Macroscopic wettability based on an interfacial jump condition
}

AUTHOR(S):

Yonemoto, Yukihiro; Kunugi, Tomoaki

CITATION:

Yonemoto, Yukihiro ... [et al]. Macroscopic wettability based on an interfacial jump condition. PHYSICAL REVIEW E 2010, 81(5): 056310.

ISSUE DATE:

2010

URL:

http://hdl.handle.net/2433/148381

RIGHT:

(C) 2010 The American Physical Society 


\title{
Macroscopic wettability based on an interfacial jump condition
}

\author{
Yukihiro Yonemoto $^{1, *}$ and Tomoaki Kunugi ${ }^{2}$ \\ ${ }^{1}$ Department of Applied Electronics, Faculty of Industrial Science and Technology, Tokyo University of Science, \\ Yamasaki 2641, Noda, Chiba 278-8510, Japan \\ ${ }^{2}$ Department of Nuclear Engineering, Kyoto University, Yoshida, Sakyo, Kyoto 606-8501, Japan \\ (Received 8 September 2009; revised manuscript received 15 December 2009; published 12 May 2010)
}

\begin{abstract}
Young's equation, describing an interfacial equilibrium condition of a liquid droplet on a smooth solid surface, raises issues concerning the existence of a sine term which has not yet been resolved theoretically and continues to be discussed to the present day. From a thermodynamics viewpoint, the equilibrium condition arises by minimizing the total free energy of the system while intensive parameters are kept constant. In the derivation, variations in the virtual work in both horizontal and vertical directions of the droplet on the smooth solid are considered. From a hydrodynamics viewpoint, there is a momentum jump condition at the gas-liquid interface that is derived based on a mechanical balance. Using standard mathematical procedures such as Stokes' theorem and differential geometry, a test volume is considered across the interface between two continuous phases from which the jump condition is derived. In the present paper, Young's equation is revisited from the point of view of the momentum jump condition at the two-phase interface and a modified Young's equation is derived. The analytical solution derived from the modified Young's equation is then used to compare theory with experimental data. The line tension and contact angle for a lens droplet are also discussed on the basis of this model.
\end{abstract}

DOI: 10.1103/PhysRevE.81.056310

\section{INTRODUCTION}

Young's equation [1] is a very important relation in the physics of liquid droplets on solid surfaces [2], forming the basis for quantifying wetting phenomena. It has been the subject of study for over 200 years since its derivation by Young in 1805 . Generally speaking, it has been stated that this equation is incomplete as an equilibrium condition because of the lack of a $\sin \theta$ term ( $\theta$, equilibrium contact angle).

Young's equation is derived mainly using a thermodynamic approach [3-6]. In the derivation, the Helmholtz free energy or the grand thermodynamic potential of the system is minimized by considering the variation in the virtual work of a droplet on a solid surface. Virtual work performed on the droplet comprises two contributions: a tangential variation at the contact line parallel to the solid surface and a normal variation perpendicular to the surface of the droplet.

Boruvka and Neumann derived the generalized Young's equation and the Young-Laplace equation based on differential geometry and thermodynamics $[7,8]$. In the derivation, the concept of line tension first described by Gibbs [9] was considered. In complete analogy to the accepted thermodynamic definitions of surface tension over a two-dimensional surface, line tension is defined as the free energy per unit length of a three-phase contact line [10,11]. By taking the line tension into account, an additional term appears in the generalized Young's equation.

Since the derivation of Young's equation, a number of modified or generalized Young's equations have been derived based on a thermodynamic approach without the virtual work variation $[12-14]$ or a lattice model $[15,16]$. How-

\footnotetext{
*Corresponding author; yonemoto@te.noda.tus.ac.jp
}

PACS number(s): 47.55.np, 68.08.Bc, 47.55.dr, 47.61.Jd

ever, the existence of the $\sin \theta$ term in Young's equation has not been solved theoretically.

In hydrodynamics, there are some studies in which the gas-liquid interfacial jump condition is derived based on the mechanical force balance $[17,18]$. This jump condition is a macroscopic scale condition at the interface in a two-phase system that consists of two continuous phases because the interface has no thickness and is characterized by the mean curvature. The jump condition is derived using Stokes' theorem, Gauss' divergence theorem, and differential geometry [19]. In these theorems, the test volume is considered across the interface between two continuous phases. This means that the derivation of the jump condition is performed by volume and surface integrations. In this concept, the interface is characterized by the curvature. The interface is thus assumed to be a Gibbs surface in the jump condition. When we consider the droplet on the solid surface, the jump condition at the interface must satisfy the mechanical equilibrium condition at the contact line via the contact angle.

In the present paper, Young's equation is reconsidered and a modified Young's equation is derived by applying the interfacial jump condition to a three-phase interface. Here, in the strict sense, the surface tension of a solid is different from that of a liquid with an additional surface stress [20-24]. Although such a contribution plays a significant role in wettability, especially on the microscopic scale [25], we assume that the solid can be defined by an "apparent" surface tension as the present paper focuses on macroscopic wettability. Thus, the solid surface tension is treated as constant in the present paper. In the derivation of Young's equation, it is assumed that the interface is the Gibbs dividing surface (mathematical surface). The contact line is taken to be a simple border line of three-phases. Thus, Young's equation is derived without the need to define the line tension. To validate our present model, the analytical solution derived from the modified Young's equation is compared with experimen- 


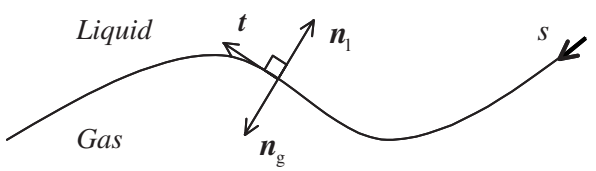

FIG. 1. Interfacial coordinates at the gas-liquid interface.

tal data $[26,27]$. In addition, to establish greater generality, the equilibrium conditions for a lens droplet are derived in a similar to the derivation of the modified Young's equation.

\section{MOMENTUM JUMP CONDITION AT THE INTERFACE}

In general, interfacial phenomena are complex and are usually interpreted as discontinuous problems. Interfaces separate two continuous equilibrium phases. When the curvature radius is much larger than the thickness of an interface, the equilibrium force balance at the interface is given by the following equation expressed in terms of interfacial coordinate system as defined in Fig. 1:

$$
\begin{aligned}
\dot{\boldsymbol{\pi}}_{g}+ & \dot{\boldsymbol{\pi}}_{l}-\left\{\left(-P_{g}\right) \boldsymbol{n}_{g}+\boldsymbol{\tau}_{g} \cdot \boldsymbol{n}_{g}\right\}-\left\{\left(-P_{l}\right) \boldsymbol{n}_{l}+\boldsymbol{\tau}_{l} \cdot \boldsymbol{n}_{l}\right\}-2 H \sigma \boldsymbol{n}_{g} \\
& -\frac{d \sigma}{d s} \boldsymbol{t}=0
\end{aligned}
$$

wherein the subscripts $l$ and $g$ signify liquid and gas phase, respectively. This equation is called the momentum jump condition at the interface. The symbols $\sigma[\mathrm{N} / \mathrm{m}], P_{k}(k=g, l)$ $\left[\mathrm{N} / \mathrm{m}^{2}\right]$, and $s[\mathrm{~m}]$ are the surface tension coefficient, pressure, and the coordinate along the interface, respectively. The mean curvature is denoted by the symbol $H\left(H=\left(\kappa_{1}+\kappa_{2}\right) / 2\right.$ with principal curvatures $\kappa_{1}$ and $\left.\kappa_{2}[1 / \mathrm{m}]\right)$. The bold symbols $\boldsymbol{n}_{k}[-], \boldsymbol{t}[-]$, and $\boldsymbol{\tau}_{k}\left[\mathrm{~N} / \mathrm{m}^{2}\right]$ are the unit normal, tangential vector, and shear stress, respectively. The symbol $\pi_{k}[\mathrm{~kg} / \mathrm{ms}]$ (with $\dot{\pi}_{k}\left[\mathrm{~N} / \mathrm{m}^{2}\right]$ the temporal differentiation of $\boldsymbol{\pi}_{k}$ ) then denotes the term related to the mass transfer, such as evaporation or condensation, through the interface.

\section{CONVENTIONAL YOUNG'S EQUATION}

Consider a droplet of liquid on a smooth solid substrate as shown in Fig. 2. The solid is insoluble in the liquid; its surface is homogeneous, continuous, and isotropic. In this ideal context, one of the most widely used equations in surface science is the following:

$$
\sigma_{g s}=\sigma_{\mathrm{lg}} \cos \theta+\sigma_{s l} .
$$

This equation is the classical Young's equation. The contact angle $(\theta)$ is related to the surface tensions of the liquid-gas, solid-gas, and solid-liquid interfaces. Generally, Eq. (2) is

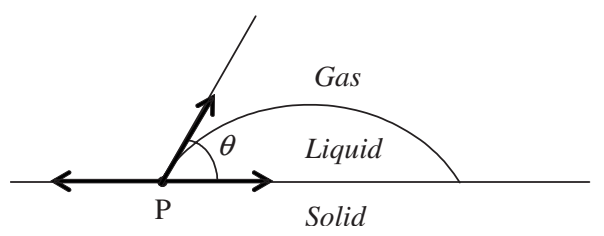

FIG. 2. Contact angle: solid/liquid/gas.

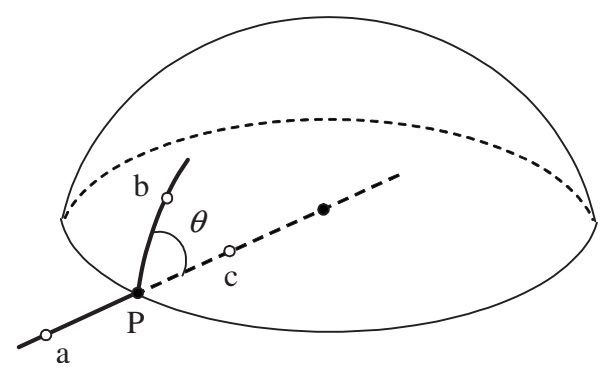

FIG. 3. Contact angle of a three-dimensional droplet on a solid substrate.

derived from thermodynamic considerations such as the virtual work variation at a contact line of the droplet. Equation (2) is supported by the classic works of Wenzel, Cassie, and Baxter who established that not only surface energy but also the solid surface roughness are factors that determine wettability [28]. Young's equation, including Young's equation combined with the Wenzel and Cassie-Baxter models, has been useful in chemical and industrial applications. However, in a strict sense, Young's equation is only valid for a two-dimensional droplet.

Boruvka and Neumann derived the generalized Young's equation from considerations of both differential geometry and thermodynamics [7]:

$$
\sigma_{g s}=\sigma_{\text {lg }} \cos \theta+\sigma_{s l}+\tau_{\text {line }} \kappa .
$$

In this equation, $\kappa[1 / \mathrm{m}]$ is the curvature calculated by the radius, $R[\mathrm{~m}]$, of the contact circle on the solid surface and $\tau_{\text {line }}[\mathrm{J} / \mathrm{m}]$ is the line tension. In addition to Eq. (3), they derived a generalized Young-Laplace equation in which both the mean and Gaussian curvatures are considered. However, they treated the normal and tangential equilibrium conditions at the contact line independently.

\section{CONSIDERATION OF YOUNG'S EQUATION}

\section{A. Derivation of Young's equation}

In an ideal system, a droplet on a solid substrate is axisymmetric. Figure 3 shows a general perspective view of a three-phase interface for an axisymmetric droplet on a solid surface. A radial cross section is shown in Fig. 2. The force balance at a point $P$ on the contact line is considered based on this system. For the sake of simplicity, mass transfer is not considered in the present treatment. We start the derivation using Eq. (1) without mass transfer which becomes

$-\left\{\left(-P_{g}\right) \boldsymbol{n}_{g}+\boldsymbol{\tau}_{g} \cdot \boldsymbol{n}_{g}\right\}-\left\{\left(-P_{l}\right) \boldsymbol{n}_{l}+\boldsymbol{\tau}_{l} \cdot \boldsymbol{n}_{l}\right\}-2 H \sigma \boldsymbol{n}_{g}-\frac{d \sigma}{d s} \boldsymbol{t}=0$.

The force balance criterion at the contact line is found by considering three interfacial line segments $P-a, P-b$, and $P-c$ forming a plane $a-b-c$ containing $P$ as illustrated in Fig. 3. We establish the two-phase interfacial coordinate system for each interface as shown in Fig. 4. Point $P$ is the origin for all interfacial coordinates. Figure 4(a) presents definitions of base vectors of the gas-solid interface. The axis coordinate $s_{a}$ 


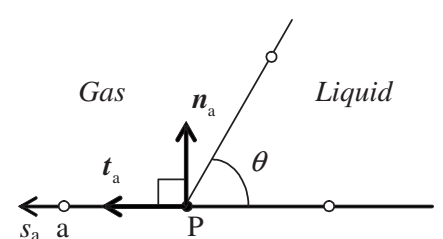

(a)

Solid
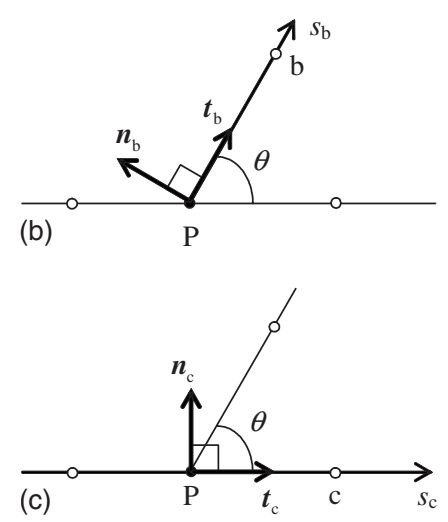

FIG. 4. Definition of base vector on a three phase line. (a) Base vector on $s_{a}$ axis $\left(P-a\right.$ line). (b) Base vector on $s_{b}$ axis $(P-b$ line). (c) Base vector on $s_{c}$ axis $(P-c$ line).

defines length on the $P$ - $a$ line segment. The direction from point $P$ to point a is the positive direction. The unit normal and tangential base vectors $\boldsymbol{n}_{a}$ and $\boldsymbol{t}_{a}$ are defined at $P$. Similar coordinate systems are defined for the other two interfaces as depicted in Figs. 4(b) and 4(c). The momentum jump conditions at each interface are defined based on each pair of base vectors.

To consider the force balance condition at the contact line where the three phases coexit, we need the connection among the three momentum jump conditions. We therefore express the jump condition as follows:

$$
\begin{aligned}
\boldsymbol{F}_{i}= & -\left(-P_{A_{i}} \boldsymbol{n}_{A_{i}}+\boldsymbol{n}_{A_{i}} \cdot \boldsymbol{\tau}_{A_{i}}\right)-\left(-P_{B_{i}} \boldsymbol{n}_{B_{i}}+\boldsymbol{n}_{B_{i}} \cdot \boldsymbol{\tau}_{B_{i}}\right)-\boldsymbol{n}_{A_{i}} 2 H_{i} \sigma_{i} \\
& -\boldsymbol{t}_{i} \frac{d \sigma_{i}}{d s_{i}},
\end{aligned}
$$

where the subscript $i(i=a, b, c)$ specifies the interface under consideration. If $i=a$, then $A=s$ and $B=g$ in Eq. (5). In the same way, if $i=b$ or $c$, then $(A, B)$ is $(l, g)$ or $(s, l)$, respectively. (Here we use the standard abbreviations: $g=$ gas, $s$ =solid, $l=$ liquid). For example, the symbol $P_{l_{b}}$ means the pressure of the liquid side of segment $P-b$. These forces balance out at the contact line of the point $P$. The force balance condition is expressed by the Neumann triangle:

$$
\boldsymbol{F}=\boldsymbol{F}_{a}+\boldsymbol{F}_{b}+\boldsymbol{F}_{c} .
$$

This force $\boldsymbol{F}\left[\mathrm{N} / \mathrm{m}^{2}\right]$ is the resultant force at the contact line. If the equilibrium condition is satisfied at the contact line, this force is zero.

From the above discussion, the force balance at the contact line can be represented using Eqs. (5) and (6). However, in a strict sense, we cannot discuss the complete equilibrium condition at the contact line because the base vectors as de- fined on each interface are independent. That is, another relation that connects the base vectors of the jump conditions is needed. This relation is obtained by applying the rotation of the base vectors. Thus, the base vectors of segments $P-b$ and $P-c$ are correlated with those of $P-a$. When the arbitrary coordinate $x-y$ in which the normal and tangential base vectors $\boldsymbol{n}$ and $\boldsymbol{t}$ are defined rotates in an anticlockwise direction about $\theta[\mathrm{deg}]$, the rotated coordinate $x^{\prime}-y^{\prime}$ in which base vectors $\boldsymbol{n}^{\prime}$ and $\boldsymbol{t}^{\prime}$ are defined is obtained as follows:

$$
\left\{\begin{array}{l}
\boldsymbol{t}^{\prime}=\boldsymbol{t} \cos \theta+\boldsymbol{n} \sin \theta \\
\boldsymbol{n}^{\prime}=-\boldsymbol{t} \sin \theta+\boldsymbol{n} \cos \theta
\end{array}\right.
$$

By using this relation, the base vectors defined on the $P-b$ segment become

$$
\left\{\begin{array}{l}
\boldsymbol{t}_{b}=-\boldsymbol{t}_{a} \cos \theta+\boldsymbol{n}_{a} \sin \theta \\
\boldsymbol{n}_{b}=\boldsymbol{t}_{a} \sin \theta+\boldsymbol{n}_{a} \cos \theta
\end{array}\right.
$$

where we use the relation between $\boldsymbol{t}_{c}$ and $\boldsymbol{t}_{a}$ as follows:

$$
\left\{\begin{array}{l}
\boldsymbol{t}_{a}=-\boldsymbol{t}_{c} \\
\boldsymbol{n}_{a}=\boldsymbol{n}_{c} .
\end{array}\right.
$$

After some algebra, substitution of Eqs. (5), (8), and (9) into Eq. (6) yields

$$
\begin{aligned}
\boldsymbol{F}= & \left\{\left(P_{g_{a}}-P_{s_{a}}\right)+\left(P_{g_{b}}-P_{l_{b}}\right) \cos \theta+\left(P_{l_{c}}-P_{s_{c}}\right)+\left(2 H_{a} \boldsymbol{\sigma}_{a}\right.\right. \\
& \left.\left.+2 H_{b} \sigma_{b} \cos \theta+2 H_{c} \sigma_{c}-\frac{d \sigma_{b}}{d s_{b}} \sin \theta\right)\right\} \boldsymbol{n}_{a}+\boldsymbol{t}_{a} \cdot\left(\boldsymbol{\tau}_{l_{b}}\right. \\
& \left.-\boldsymbol{\tau}_{g_{b}}\right) \sin \theta+\left\{\left(P_{g_{b}}-P_{l_{b}}\right) \sin \theta+\left(2 H_{b} \sigma_{b} \sin \theta-\frac{d \sigma_{a}}{d s_{a}}\right.\right. \\
& \left.\left.+\frac{d \sigma_{b}}{d s_{b}} \cos \theta+\frac{d \sigma_{c}}{d s_{c}}\right)\right\} \boldsymbol{t}_{a}+\boldsymbol{n}_{a} \cdot\left(\boldsymbol{\tau}_{l_{b}}-\boldsymbol{\tau}_{g_{b}}\right) \cos \theta+\boldsymbol{n}_{a} \cdot\left\{\left(\boldsymbol{\tau}_{s_{a}}\right.\right. \\
& \left.\left.-\boldsymbol{\tau}_{g_{a}}\right)+\left(\boldsymbol{\tau}_{s_{c}}-\boldsymbol{\tau}_{l_{c}}\right)\right\} .
\end{aligned}
$$

In this equation, we assume static conditions and therefore the shear stress can be ignored. Considering the equilibrium state in Eq. (10), the coefficients of each base vectors $\boldsymbol{n}_{a}$ and $\boldsymbol{t}_{a}$ are set equal to zero. Thus, we obtain the relation from the coefficient of normal vector $\boldsymbol{n}_{a}$ as follows:

$$
\begin{aligned}
0= & \left(P_{g_{a}}-P_{s_{a}}\right)+\left(P_{g_{b}}-P_{l_{b}}\right) \cos \theta+\left(P_{l_{c}}-P_{s_{c}}\right) \\
& +\left(2 H_{a} \sigma_{a}+2 H_{b} \sigma_{b} \cos \theta+2 H_{c} \sigma_{c}-\frac{d \sigma_{b}}{d s_{b}} \sin \theta\right)
\end{aligned}
$$

and that from the tangential vector $\boldsymbol{t}_{a}$ as follows:

$$
\begin{aligned}
0= & \left(P_{g_{b}}-P_{l_{b}}\right) \sin \theta+\left(2 H_{b} \sigma_{b} \sin \theta-\frac{d \sigma_{a}}{d s_{a}}+\frac{d \sigma_{b}}{d s_{b}} \cos \theta\right. \\
& \left.+\frac{d \sigma_{c}}{d s_{c}}\right) .
\end{aligned}
$$

We now integrate Eqs. (11) and (12) over the arbitrary infinitesimal area $d A=d w d s_{a}$ as shown in Fig. 5. The symbol $d w$ represents the element of the contact line. These integrated 


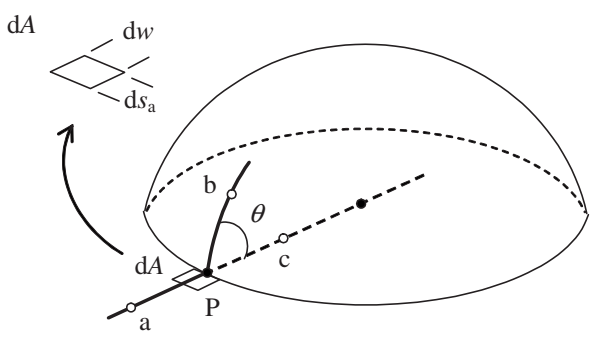

FIG. 5. Small area on $s_{a}$ axis.

equations represent force balances in the normal and tangential directions. In this paper, we focus on the local force balance. Thus, we assume that $d s_{a}=d s_{b}=d s_{c}=d s$. The integration of Eq. (11) over the area $d A$ yields

$$
\begin{aligned}
0= & \iint_{A}\left\{\left(P_{g_{a}}-P_{s_{a}}\right)+\left(P_{g_{b}}-P_{l_{b}}\right) \cos \theta+\left(P_{l_{c}}-P_{s_{c}}\right)\right. \\
& \left.+2 H_{a} \sigma_{a}+2 H_{b} \sigma_{b} \cos \theta+2 H_{c} \sigma_{c}-\frac{d \sigma_{b}}{d s_{a}} \sin \theta\right\} d s_{a} d w .
\end{aligned}
$$

In this equation, we integrate $d s_{a}$ arbitrarily from 0 to small finite length $l_{s}[\mathrm{~m}]$. The integration of the last term on the right-hand side of Eq. (13) gives

$$
\int_{0}^{l_{s}} \frac{d \sigma_{b}}{d s_{a}} \sin \theta d s_{a}=\sin \theta \int_{\sigma_{b}(0)}^{\sigma_{b}\left(l_{s}\right)} d \sigma_{b}=\sigma_{b} \sin \theta .
$$

The calculation of the surface tension coefficient $\sigma_{b}$ depends on its functional relation in the problem. If there is no contamination at the interface, $\sigma_{b}$ is constant at the gas-liquid interface. Moreover, it is assumed that the curvature terms are independent of the integration because the force acts locally along the contact line; that is, this assumption simply means that integration over $d s_{a}$ reduces to multiplying by length $l_{s}[\mathrm{~m}]$. Finally, the following normal equilibrium condition is obtained:

$$
\begin{aligned}
0= & \left\{\left(P_{g_{b}}-P_{l_{b}}\right) \cos \theta+\left(P_{g_{a}}-P_{s_{a}}\right)+\left(P_{l_{c}}-P_{s_{c}}\right)\right. \\
& \left.+2 H_{b} \sigma_{b} \cos \theta+2 H_{a} \sigma_{a}+2 H_{c} \sigma_{c}\right\} l_{s}-\sigma_{b} \sin \theta .
\end{aligned}
$$

Similarly, we obtain the tangential equilibrium condition at the contact line from Eq. (12) as follows:

$$
0=\left\{\left(P_{g_{b}}-P_{l_{b}}\right) \sin \theta+2 H_{b} \sigma_{b} \sin \theta\right\} l_{s}-\sigma_{a}+\sigma_{b} \cos \theta+\sigma_{c} .
$$

Equation (15) is generally neglected because the normal force balances out on the solid surface. Equation (16) is Young's equation with additional terms and differs from the conventional Young's equation [Eq. (2)]. A detailed description of the surface tension acting around a three-phase contact line is shown in Fig. 6. The surface tension related to the curvature at the gas-liquid interface is decomposed into normal and tangential components on the solid surface in Fig. 6(a). From this description, the force $2 H_{b} \sigma_{b} \sin \theta$ can be readily understood as the tangential component of surface tension $2 H_{b} \sigma_{b}$ acting normal to the droplet surface at the
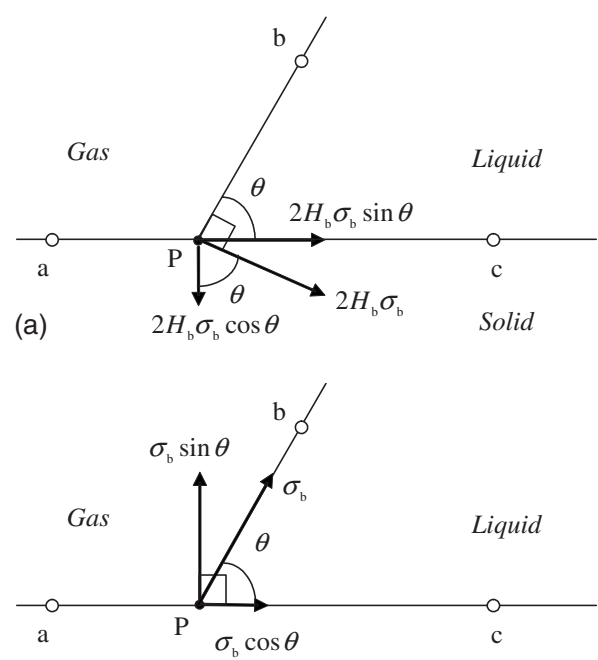

(b)

Solid

FIG. 6. Schematic of the term $\sigma_{b} \sin \theta$ at the contact line. [Decomposition of surface tension in the (a) normal and (b) tangential direction with respect to the gas-liquid interface.]

contact line on the solid surface. Furthermore, as shown in Fig. 6(b), the force $\sigma_{b} \sin \theta$ is directed normal to the solid surface at the contact line.

While Young's equation is true in only planar geometry, Eqs. (15) and (16) can be applied in a nonplanar geometry, such as droplets on a sphere or a curved plate.

\section{B. Derivation of analytical solution}

In this section, an analytical solution is derived from our modified Young's equation, a validation of which is given by comparison with reported experimental data [26,27]. The tangential equilibrium condition given by Eq. (16) is considered primarily in this derivation. As in Eq. (3), Boruvka and Neumann derived the generalized Young's equation based on differential geometry and a thermodynamic approach. Amirfazli and co-workers studied and evaluated the line tension based on this equation [26,29-31]. In their experiments, the drop-size dependence of contact angles was studied using sessile droplets on several different substrates. The contact angle was measured to within $20^{\circ}$, and liquids such as heptane and octane were employed. The measured data were evaluated based on the following procedure.

For a very large droplet (i.e., in the limit $\tau_{\mathrm{line}} \kappa / \sigma_{\mathrm{lg}} \ll 1$ ), the last term in the right-hand side of Eq. (3) vanishes and Young's equation is recovered as

$$
\sigma_{g s}=\sigma_{\mathrm{lg}} \cos \theta_{\infty}+\sigma_{s l},
$$

where $\theta_{\infty}$ is the contact angle corresponding to an infinitely large drop. Combining Eqs. (3) and (17) leads to

$$
\cos \theta=\cos \theta_{\infty}-\frac{\tau_{\text {line }}}{\sigma_{\text {lg }}} \frac{1}{R},
$$

which was treated by Amirfazli et al. [26] as having a linear dependence between $\cos \theta$ and $1 / R$. After performing an experiment wherein the drop radius, $R$, is changed while the contact angle is measured, one can obtain a plot of $\cos \theta$ vs 


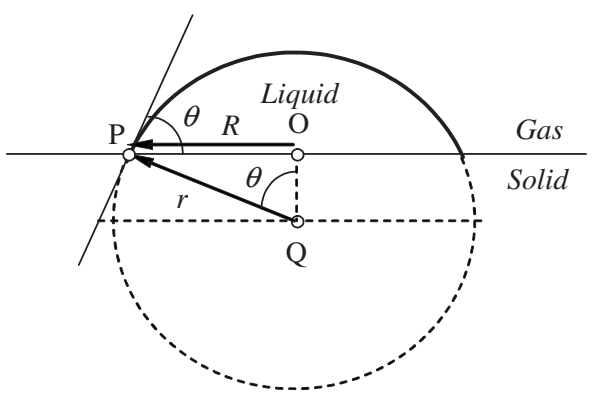

FIG. 7. Ideal droplet on a solid substrate.

$1 / R$. The line tension, $\tau_{\text {line }}$, can be determined from the slope $-\tau_{\text {line }} / \sigma_{\text {lg }}$ of the plot, and the infinite radius contact angle $\theta_{\infty}$ can be obtained from the intercept. Based on these results, the concept of line tension is discussed. Amirfazli and Neumann recently reviewed the status of the three-phase line tension, including a comprehensive bibliography on line tension [32]. In the review, it is reported that there is no consensus regarding line tension. The reported experimentally measured line tensions vary from approximately $10^{-9}$ to $10^{-6}[\mathrm{~J} / \mathrm{m}]$ and have either positive or negative sign. Moreover, it seems that line tension decreases as the contact angle decreases and likely vanishes at complete wetting. The detailed mechanism for this behavior is unknown.

We now carry out modeling of Young's equation and derive the analytical solution. In the present paper, it is assumed that the droplet on the solid surface is very small $\left(P_{g_{b}} \approx P_{l_{b}}\right)$. Thus, using Young's equation without the pressure term of Eq. (16), it can be expressed simply as

$$
\sigma_{a}=2 H_{b} \sigma_{b} l_{s} \sin \theta+\sigma_{b} \cos \theta+\sigma_{c},
$$

where the mean curvature $H_{b}$ is defined on the gas-liquid interface at the contact line. In a manner similar to that taken in the derivation of Eq. (18), the first term in the right-hand side of Eq. (19), $2 H_{b} \sigma_{b} \sin \theta$, vanishes for a very large droplet, and Young's equation is recovered in the form of Eq. (17). By taking Eq. (17) into account, Eq. (19) becomes

$$
\cos \theta=\cos \theta_{\infty}-2 H_{b} l_{s} \sin \theta .
$$

In Eq. (18), the curvature is based on the radius of the contact circle on the solid surface. Therefore, the mean curvature $H_{b}$ is associated with the curvature characterized by the contact circle based on the following assumption.

We again consider the ideal system of an axisymmetric droplet on a solid surface as shown in Fig. 2; its volume as a cap of a sphere is indicated in Fig. 7. The radius of the sphere is $r[\mathrm{~m}]$ and the radius of the contact circle is $R$. Given the contact angle $\theta$, the relation between $r$ and $R$ is given by

$$
R=r \cos \theta \text {. }
$$

Considering a real situation, Eq. (21) no longer holds due to the imperfect condition at the solid surface and the properties of the droplet. Therefore, we incorporate a parameter $C_{0}[-]$ into Eq. (21) as

$$
R=C_{0} r \sin \theta .
$$

Using this equation, the mean curvature $H_{b}$ in Eq. (20) is calculated as

$$
H_{b}=\frac{1}{2}\left(\frac{1}{r_{1}}+\frac{1}{r_{2}}\right)=\frac{1}{r}=\frac{C_{0} \sin \theta}{R},
$$

where $1 / r_{1}$ and $1 / r_{2}$ are the principal curvatures on the gasliquid interface at the contact line. Finally, substituting Eq. (23) into Eq. (20) gives

$$
R=-\frac{2 C_{0} l_{s} \sin ^{2} \theta}{\cos \theta-\cos \theta_{\infty}} .
$$

This equation represents the relation between the radius of the contact circle and the contact angle that can be used to evaluate experimental data. Here, the additional term $\left(2 H_{b} \sigma_{b} l_{s} \sin \theta\right)$ in Eq. (19) can be rewritten as

$$
\begin{aligned}
2 H_{b} \sigma_{b} l_{s} \sin \theta & =\frac{2}{r} \sigma_{b} l_{s} \sin \theta=\frac{2 C_{0} \sigma_{b} l_{s} \sin ^{2} \theta}{R} \\
& =2 C_{0} \kappa \sigma_{b} l_{s} \sin ^{2} \theta .
\end{aligned}
$$

Comparing Eq. (19) with Eq. (3) after applying Eq. (25) into Eq. (19), the following equation is obtained:

$$
\tau_{\text {line }}=2 C_{0} \sigma_{b} l_{s} \sin ^{2} \theta .
$$

In this paper, the line tension is not explicitly defined. However, if Eq. (3) is satisfied, Eq. (26) could be used to define the line tension. This equation indicates its dependence on the contact angle [32].

In Eq. (24), the parameter $C_{0}$ is estimated as

$$
C_{0}=C_{1} \sigma_{b},
$$

where the parameter $C_{1}[\mathrm{~m} / \mathrm{N}]$ is determined by experiment. Many models may be assumed for $C_{0}$. The choice of Eq. (27) follows from the fact that the wettability depends on the combination of droplets and solid surfaces if there is no contamination on the solid surfaces and in the droplets.

\section{RESULTS AND DISCUSSION}

In the previous section, we derived the analytical solution of Young's equation. Here, we use Eq. (24) and compare its analytical solution with reported experimental data. In the comparison, the experimental data of Amirfazli et al. and Duncan et al. [26,27] are used. An arbitrary parameter $C_{2}$ $=C_{1} l_{s}\left[\mathrm{~m}^{2} / \mathrm{N}\right]$ is defined as a fitting parameter in Eq. (24).

Figure 8 shows the results of the drop-size dependence on the contact angles [26]. The liquids used are heptane and octane. The surface tension coefficients of heptane and octane are 19.9 and $21.4\left[\mathrm{~mJ} / \mathrm{m}^{2}\right]$ at $22 \pm 1{ }^{\circ} \mathrm{C}$, respectively. In Eq. (24), values of $C_{2}$ for heptane and octane are taken as $1.53 \times 10^{-2}$ and $1.04 \times 10^{-2}$, respectively. Amirfazli et al. estimate the infinite contact angle by linear interpolation $(\cos \theta$ vs $\kappa$ ) as $\theta_{\infty}=4.0^{\circ}$ in heptane and $\theta_{\infty}=6.7^{\circ}$ in octane. In Fig. 8 , the dashed line is the result of Eq. (24). The analytical solution shows fair agreement with the experimental data. However, the analytical solution for heptane is slightly dif- 


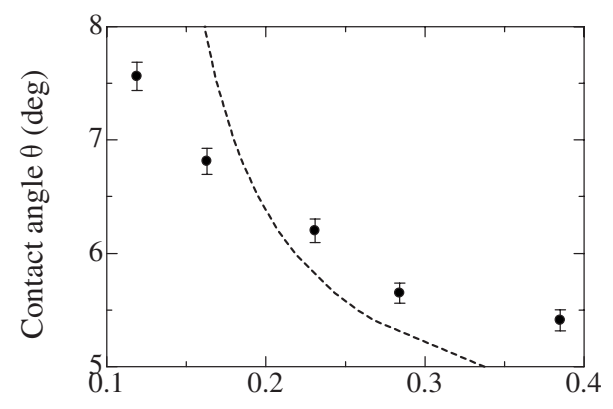

(a) Radius of droplet on the substrate $(\mathrm{cm})$

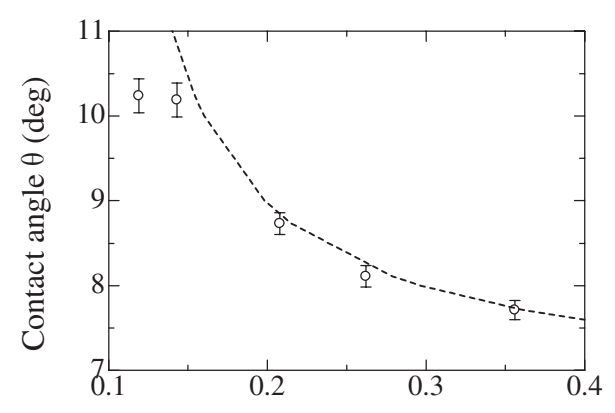

(b) Radius of droplet on the substrate $(\mathrm{cm})$

FIG. 8. Experimental data with respect to droplet radius and contact angle (adapted from Ref. [26]). (a) Droplet radius vs contact angle of heptane $\left(C_{2}=1.53 \times 10^{-2}\left[\mathrm{~m}^{2} / \mathrm{N}\right], \theta_{\infty}=4.0^{\circ}\right)$. (b) Droplet radius vs contact angle of octane $\left(C_{2}=1.04 \times 10^{-2}\left[\mathrm{~m}^{2} / \mathrm{N}\right], \theta_{\infty}\right.$ $=6.7^{\circ}$ ).

ferent from the experimental data. It is conjectured that the difference is related to the use of the estimated value of $\theta_{\infty}$ from the linear interpolation in the analytical solution. Figure 9 shows the best fit to the experimental data for heptane, which is obtained with an estimated $\theta_{\infty}$ of $4.8^{\circ}$ and $C_{2}$ value of $1.04 \times 10^{-2}$ as adopted for octane. The analytical solution reproduces the experimentally observed relationship well using these parameter values.

Figure 10 shows the results of the analytical solution of Eq. (24) and the experimental data reported by Duncan et al. [27]. The experiment was performed using dodecane on a substrate of a FC-721 dip-coated onto mica (FC-721, 3M). The surface tension of dodecane is $25.44\left[\mathrm{~mJ} / \mathrm{m}^{2}\right]$. It is found

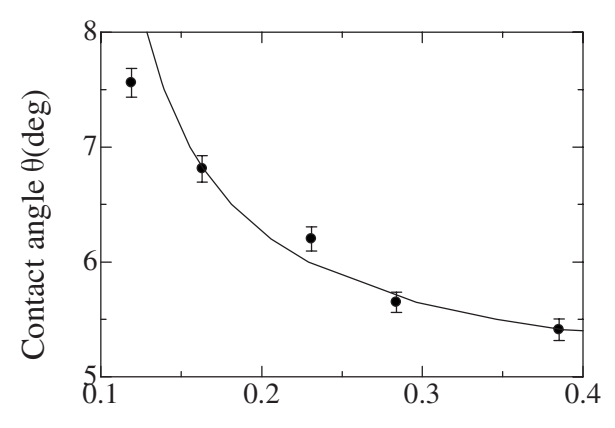

Radius of droplet on the substrate $(\mathrm{cm})$

FIG. 9. Droplet radius vs contact angle of heptane $\left(C_{2}=1.04\right.$ $\left.\times 10^{-2}\left[\mathrm{~m}^{2} / \mathrm{N}\right], \quad \theta_{\infty}=4.8^{\circ}\right)$.

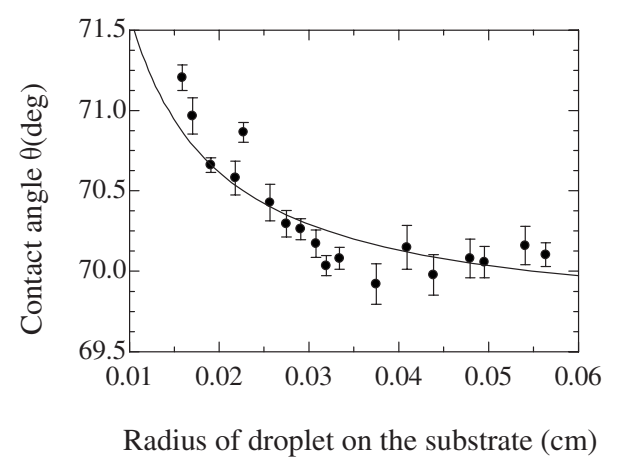

FIG. 10. Droplet radius vs contact angle of dodecane $\left(C_{2}=7.0\right.$ $\left.\times 10^{-5}\left[\mathrm{~m}^{2} / \mathrm{N}\right], \theta_{\infty}=69.65^{\circ}\right)($ adapted from Ref. [27]).

that the analytical solution agrees well with experimental data. In this analysis, $C_{2}=7.0 \times 10^{-5}$ and $\theta_{\infty}=69.65^{\circ}$. A similar trend between droplet radius and contact angle is observed in other experiment [33].

Figure 11 shows the result for the predicted line tension using Eq. (26). The line tensions of heptane and octane are shown in Fig. 11. This result shows that the line tension depends on the contact angle and its value vanishes when the contact angle approaches zero.

The present model can explain the experimental data reported by some authors. It appears that the line tension is characterized by the surface tension coefficient of the droplet and the geometry (contact angle, etc.) near the contact line. Thus the conventional line tension may not be a single thermodynamic value defined at the contact line. The parameter $C_{0}$ may depend on the physical property of the liquid and the substrate. That is, we may be able to interpret $2 C_{0} l_{s} \sigma_{b}$ as the line tension in Eq. (26). The right-hand side of Eq. (25) may involve the line tension $2 C_{0} l_{s} \sigma_{b}$, the curvature $\kappa$ of the contact circle, and the contact angle $\theta$.

\section{CONTACT ANGLE FOR A LENS DROPLET}

We now consider the contact angle for a lens droplet. The basic idea is the same as the derivation of the modified Young's equation. We consider the system in which there are three phases ( $\alpha, \beta$, and $\gamma$ phases) as shown in Fig. 12. The mathematical definitions are the same as in the derivation of the modified Young's equation. That is, we define the base

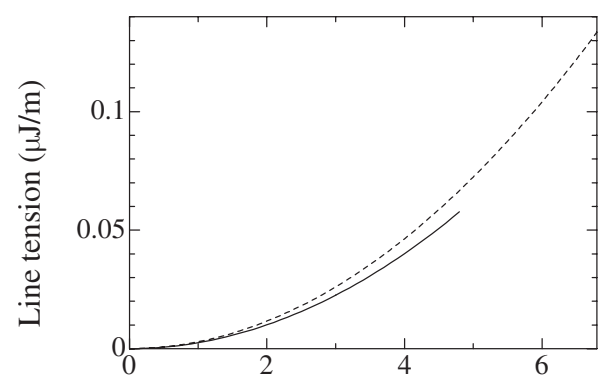

Contact angle $\theta(\mathrm{deg})$

FIG. 11. Analytical solution of Line tension of octane and heptane in Figs. 8 and 9 (solid line: heptane, dashed line: octane). 

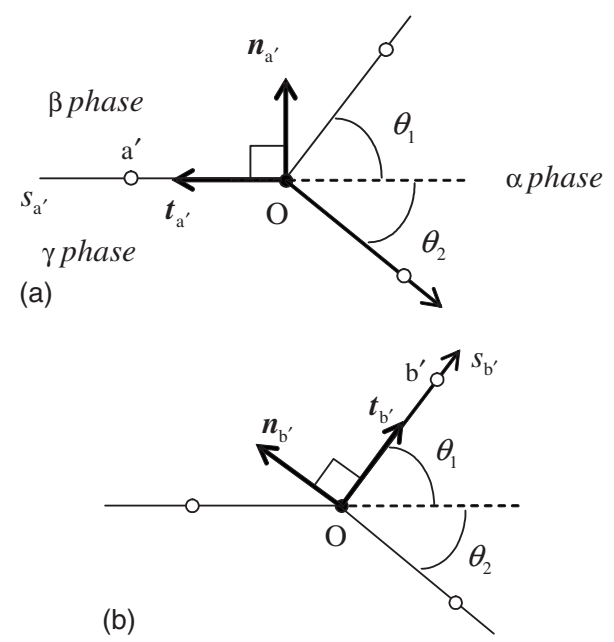

(b)

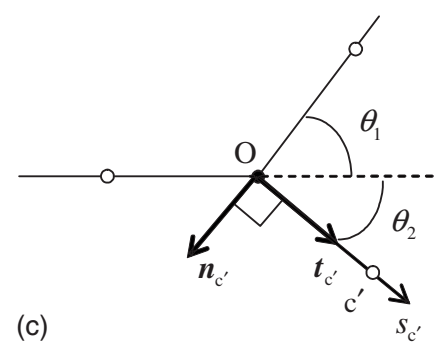

FIG. 12. Definition of base vector on three-phase line of a lens droplet. (a) Base vector on $s_{a^{\prime}}$ axis $\left(O-a^{\prime}\right.$ line). (b) Base vector on $s_{b^{\prime}}$ axis $\left(O-b^{\prime}\right.$ line $)$. (c) Base vector on $s_{c^{\prime}}$ axis $\left(O-c^{\prime}\right.$ line $)$.

vectors on each axis. Thus, the jump conditions at the interfaces are defined on each interface based on the base vectors. In this system, a lens droplet of the $\alpha$ phase is on the $\gamma$ phase. This system is ideal, i.e., insoluble, axisymmetric, and without contamination. Based on this system, we consider the force balance at the contact line of point $O$.

We apply the rotation of base vectors in this system. The base vectors of $O-b^{\prime}$ and $O-c^{\prime}$ axes are represented by using the base vectors of the $O-a^{\prime}$ axis. In a similar way as in Eq. (7), the base vectors of the $O-b^{\prime}$ axis can be expressed as

$$
\left\{\begin{array}{l}
\boldsymbol{t}_{b^{\prime}}=-\boldsymbol{t}_{a^{\prime}} \cos \theta_{1}+\boldsymbol{n}_{a^{\prime}} \sin \theta_{1} \\
\boldsymbol{n}_{b^{\prime}}=\boldsymbol{t}_{a^{\prime}} \sin \theta_{1}+\boldsymbol{n}_{a^{\prime}} \cos \theta_{1}
\end{array}\right.
$$

and the base vectors of $O-c^{\prime}$ axis as:

$$
\left\{\begin{array}{l}
\boldsymbol{t}_{c^{\prime}}=-\boldsymbol{t}_{a^{\prime}} \cos \theta_{2}-\boldsymbol{n}_{a^{\prime}} \sin \theta_{2} \\
\boldsymbol{n}_{c^{\prime}}=\boldsymbol{t}_{a^{\prime}} \sin \theta_{2}-\boldsymbol{n}_{a^{\prime}} \cos \theta_{2},
\end{array}\right.
$$

wherein $\theta_{1}>0$ and $\theta_{2}>0$. By using Eqs. (28) and (29) and using the same procedure as for the derivation of the modified Young's equation, we find the normal force balance at the contact line to be

$$
\begin{aligned}
0= & \left\{\left(P_{\beta_{a^{\prime}}}-P_{\gamma_{a^{\prime}}}\right)+\left(P_{\beta_{b^{\prime}}}-P_{\alpha_{b^{\prime}}}\right) \cos \theta_{1}+\left(P_{\alpha_{c^{\prime}}}-P_{\gamma_{c^{\prime}}}\right) \cos \theta_{2}\right. \\
& \left.+2 H_{a^{\prime}} \sigma_{a^{\prime}}+2 H_{b^{\prime}} \sigma_{b^{\prime}} \cos \theta_{1}-2 H_{c^{\prime}} \sigma_{c^{\prime}} \cos \theta_{2}\right\} l_{s} \\
& -\sigma_{b^{\prime}} \sin \theta_{1}+\sigma_{c^{\prime}} \sin \theta_{2} .
\end{aligned}
$$

Then, the tangential force balance is given by

$$
\begin{aligned}
0= & \left\{\left(P_{\beta_{b^{\prime}}}-P_{\alpha_{b^{\prime}}}\right) \sin \theta_{1}+\left(P_{\gamma_{c^{\prime}}}-P_{\alpha_{c^{\prime}}}\right) \sin \theta_{2}+2 H_{b^{\prime}} \sigma_{b^{\prime}} \sin \theta_{1}\right. \\
& \left.+2 H_{c^{\prime}} \sigma_{c^{\prime}} \sin \theta_{2}\right\} l_{s}-\sigma_{a^{\prime}}+\sigma_{b^{\prime}} \cos \theta_{1}+\sigma_{c^{\prime}} \cos \theta_{2} .
\end{aligned}
$$

The labeling is such that the subscript $\alpha_{b^{\prime}}$, for example, denotes the $\alpha$ phase side of the $b^{\prime}$ axis.

\section{CONCLUSIONS}

In this paper, Young's equation was derived based on a hydrodynamic approach. In the derivation, the mechanical equilibrium condition at the contact line of a droplet on the solid surface was considered by using the interfacial jump condition. From this consideration, we obtained the following results:

(1) The analytical solution derived from our modified Young's equation can explain the experimental data, such as the relationship between the contact angle and the radius of the contact circle.

(2) The force $\sigma_{b} \sin \theta$ arises in the normal mechanical equilibrium condition at the contact line on the solid surface.

(3) The force $2 H_{b} \sigma_{b} l_{s} \sin \theta$ arises in the tangential mechanical equilibrium condition at the contact line on the solid surface. This additional force is related to the surface tension $2 H_{b} \sigma_{b}$ normal to the droplet surface. That is, $2 H_{b} \sigma_{b} l_{s} \sin \theta$ corresponds to the tangential component of $2 H_{b} \sigma_{b}$ evaluated on the solid surface.

(4) By comparing the present model [Eq. (19)] with the generalized Young's equation [Eq. (3)], we have determined the detailed equation of the line tension, from which the line tension vanishes as the contact angle approaches zero. This means that the line tension depends on the contact angle. Eventually, the line tension is characterized by the surface tension coefficient of the droplet and the geometry near the contact line. The conventional line tension may not be a single thermodynamic value defined at the contact line. The parameter $C_{0}$ may depend on physical properties of the liquid and the substrate. That is, we may be able to interpret $2 C_{0} l_{s} \sigma_{b}$ as the line tension in Eq. (26). The right-hand side of Eq. (25) may consist of the line tension $2 C_{0} l_{s} \sigma_{b}$, the curvature $\kappa$ of the contact circle, and the contact angle $\theta$.

(5) We consider the mechanical equilibrium condition of the lens droplet by using the same concept as in the derivation of the modified Young's equation. The tangential and normal mechanical equilibrium conditions at the contact angle are derived. This means that the proposed theory can be applied to more general wetting phenomena such as droplets on curved surfaces. 
[1] T. Young, Philos. Trans. R. Soc. London, Ser. A 95, 65 (1805).

[2] P. G. de Gennes, F. Brochard-Wyart, and D. Quéré, Capillarity and Wetting Phenomena, Drops, Bubbles, Pearls, Waves (Springer, New York, 2004).

[3] D. Li and F. Y. H. Lin, Colloids Surf., A 87, 93 (1994).

[4] R. E. Johnson, J. Phys. Chem. 63, 1655 (1959).

[5] P. Roura and J. Fort, J. Colloid Interface Sci. 272, 420 (2004).

[6] P. Roura, Am. J. Phys. 73, 1139 (2005).

[7] L. Boruvka and A. W. Neumann, J. Chem. Phys. 66, 5464 (1977).

[8] J. Gaydos and A. W. Neumann, J. Colloid Interface Sci. 120, 76 (1987).

[9] J. W. Gibbs, The Scientific Papers of J. Willard Gibbs (Dover, New York, 1961).

[10] B. Widom and A. S. Clarke, Physica A 168, 149 (1990).

[11] J. S. Rowlinson and B. Widom, Molecular Theory of Capillarity (Oxford University Press, New York, 1984).

[12] J. Coninck and F. Dunlop, J. Stat. Phys. 47, 827 (1987).

[13] J. Coninck, F. Dunlop, and V. Rivasseau, Commun. Math. Phys. 121, 401 (1989).

[14] P. Letellier, A. Mayaffre, and M. Turmine, J. Colloid Interface Sci. 314, 604 (2007).

[15] D. B. Abraham and L. F. Ko, Phys. Rev. Lett. 63, 275 (1989).

[16] J. De Coninck, J. Ruiz, and S. Miracle-Solé, Phys. Rev. E 65, 036139 (2002).

[17] L. E. Scriven, Chem. Eng. Sci. 12, 98 (1960).

[18] J. M. Delhaye, Int. J. Multiphase Flow 1, 395 (1974).

[19] R. Aris, Tensors and the Basic Equations of Fluid Mechanics
(Prentice-Hall, New Jersey, 1962).

[20] R. Shuttleworth, Proc. Phys. Soc., London, Sect. A 63, 444 (1950)

[21] E. Orowan, Proc. R. Soc. London, Ser. A 316, 473 (1970).

[22] P. Tarazona and G. Navascues, Surf. Sci. 111, L733 (1981).

[23] J. S. Vermaak, C. W. Mays, and D. Kuhlmann-Wilsdorf, Surf. Sci. 12, 128 (1968).

[24] D. E. Wolf, R. B. Griffiths, and L. Tang, Surf. Sci. 162, 114 (1985)

[25] J. Koleske, Paint and Coatings Testing Manual, 14th ed. (American Society for Testing and Materials, Philadelphia, 1995).

[26] A. Amirfazli, A. Keshavarz, L. Zhang, and A. W. Neumann, J. Colloid Interface Sci. 265, 152 (2003).

[27] D. Duncan, D. Li, J. Gaydos, and A. W. Neumann, J. Colloid Interface Sci. 169, 256 (1995).

[28] S. M. Lee and T. H. Kwon, J. Micromech. Microeng. 17, 687 (2007).

[29] A. Amirfazli, D. Chatain, and A. W. Neumann, Colloids Surf., A 142, 183 (1998).

[30] A. Amirfazli, D. Y. Kwok, J. Gaydos, and A. W. Neumann, J. Colloid Interface Sci. 205, 1 (1998).

[31] A. Amirfazli, S. Hänig, A. Müller, and A. W. Neumann, Langmuir 16, 2024 (2000).

[32] A. Amirfazli and A. W. Neumann, Adv. Colloid Interface Sci. 110, 121 (2004).

[33] Y. Gu, Colloids Surf., A 181, 215 (2001). 